\section{Dicke Kinder brechen sich häufiger die Knochen}

Kinder, die für ihr Alter zu viele Pfunde auf die Waage bringen, brechen sich häufiger das Bein - vor allem Fuß und Unterschenkel - als normalgewichtige Altersgenossen. Gleich zwei Teams von Wissenschaftlern aus den USA und Italien kommen in ihren Studien zu diesem Ergebnis.

$D^{3}$ as Risiko einer Fraktur an Fuß, Knöchel, Unterschenkel oder im Kniebereich stieg in einer US-amerikanischen Untersuchung bei Kindern bereits ab einem BMI von $25 \mathrm{~kg} / \mathrm{m}^{2}(85$. bis 95. Perzentile) stetig an. Leicht übergewichtige Kinder hatten ein um $17 \%$ erhöhtes Risiko gegenüber normalgewichtigen Altersgenossen, bei extrem adipösen (BMI $\geq 30 \mathrm{~kg} / \mathrm{m}^{2}$ ) war die Wahrscheinlichkeit eines Knochenbruchs an der unteren Extremität bis einschließlich zum Knie bereits um 45 \% erhöht. Die Studie von Jeff Kessler et al. [1] beruht auf Daten von fast einer Million Kindern im Alter zwischen zwei und 19 Jahren, die an einer großen

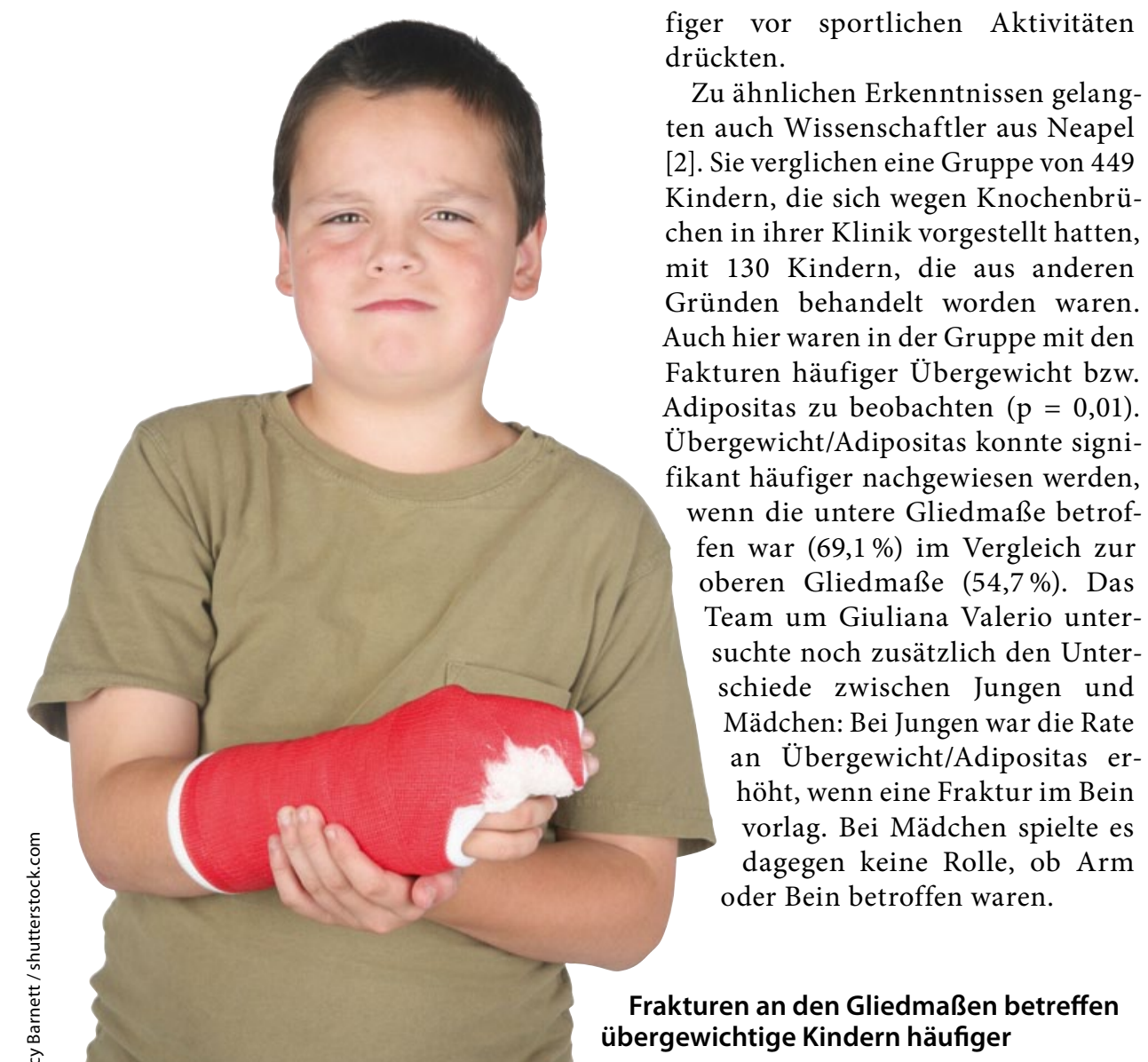

Die Forscher verglichen außerdem verschiedenen Verhaltensmuster in den beiden Gruppen mit und ohne Frakturen. Die Kinder der Gruppe mit den Knochenbrüchen waren demnach sportlich aktiver; die meisten Verletzungen zogen sie sich beim Fußballtraining zu. Andererseits war in dieser Gruppe auch der Fernsehkonsum (zwei Stunden oder mehr pro Tag) höher als in der Kontrollgruppe.

Kaiser Permanente Children's Health Study, teilgenommen hatten.

Am deutlichsten war die Assoziation zwischen Übergewicht und Frakturrisiko bei Kindern zwischen sechs und elf Jahren, aber eben nur vom Knie abwärts. Die Gefahr, sich den Oberschenkel zu brechen, war dagegen in der Gesamtkohorte überraschenderweise nur bei zu dünnen Teilnehmern im Vergleich zu normalgewichtigen erhöht (um immerhin $66 \%$ ). Übergewichtige Teenies waren im Hinblick auf Femurfrakturen deutlich weniger gefährdet als ihre normal- oder untergewichtigen Altersgenossen, dies aber wohl nur, weil sie sich tendenziell häufiger vor sportlichen Aktivitäten rückten

$\mathrm{Zu}$ ähnlichen Erkenntnissen gelangten auch Wissenschaftler aus Neapel [2]. Sie verglichen eine Gruppe von 449 Kindern, die sich wegen Knochenbrüchen in ihrer Klinik vorgestellt hatten, mit 130 Kindern, die aus anderen Gründen behandelt worden waren. Auch hier waren in der Gruppe mit den Fakturen häufiger Übergewicht bzw. Adipositas $\mathrm{zu}$ beobachten $(\mathrm{p}=0,01)$. nte signiwerden

1. Kessler J et al. Childhood obesity is associated with increased risk of most lower extremity fractures. Clin Orthop Relat Res 2012; online 5. Oktober; doi: 10.1007/s11999-012-2621-z

2. Valerio $G$ et al. Prevalence of overweight in children with bone fractures: a case control study. BMC Pediatr 2012 Oct 22; 12: 166. [Epub ahead of print]

Kommentar: Für die Studienautoren sind die Ergebnisse ein weiteres Argument dafür, dem Übergewicht bereits in jungen Jahren und frühen Stadien zu begegnen. In Deutschland gelten nach aktuellen Zahlen des Robert-Koch-Instituts 14,8\% der Kinder und Jugendlichen zwischen zwei und 17 Jahren als übergewichtig, $6,1 \%$ davon sind adipös (KIGGS Kinder und Jugend Gesundheitssurvey). Allerdings bedingt auch sportliche Aktivität ein erhöhtes Frakturrisiko. Interessant ist auch, dass übergewichtige Kinder sich seltener den Oberschenkel brechen als dünne Kinder. Aber auch diese differenzierten Ergebnisse sollten weder davon abhalten, für Normalgewicht bei Kindern und Jugendlichen zu kämpfen, noch davon, sportliche Aktivitäten zu fördern. Beides wird dazu beitragen, die langfristige Gesundheit der Kinder zu erhalten.

Dr. Martin Claßen/nz

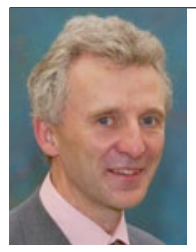

Dr. med. Martin Claßen Chefarzt der Klinik für Kinder- $u$. Jugendmedizin Klinikum Links der Weser Senator-Weßling-Str. 1 28277 Bremen 